\title{
Infecção natural por Histomonas meleagridis em pavões-indianos (Pavo cristatus)
}

\author{
Histomonas meleagridis Natural Infection in Peacocks (Pavo cristatus)
}

\author{
Ricardo Almeida da Costa ${ }^{1,2}$, Ana Paula Maurique Pereira ${ }^{1}$, Caroline da Silva Silveira ${ }^{1,2} \&$ Bruno Leite Anjos ${ }^{1}$
}

\begin{abstract}
Background: Histomonas, also known as blackhead, is a protozoan disease caused by Histomonas meleagridis (phylum Parabasalia, class Tritrichomonadea, order Tritrichomonadida, family Dientamoebidae/Protrichomonadinae) and, characterized by enteric and hepatic lesions in several species of domestic and wild gallinaceous. Among the most affected species, turkeys are those with greater morbidity and mortality. Blackhead is the second most important disease caused by protozoa in domestic poultry, affecting especially young birds and causing severe economic losses, with decrease in production, lack of uniformity of lots and costs with treatment and control. Its occurrence must be monitored, especially in large poultry exporting countries, such as Brazil. The aim of this study is to report an outbreak of histomoniasis in Indian peacocks

Case: A peacocks group created together with chickens showed clinical signs characterized by apathy, head down and bruised head, eyes closed, anorexia, yellow or pale stools and death. At necropsy, the cecum showed intense thickening of the wall with irregularity in the serous layer and abundant deposit of friable material, amorphous, yellowish color with areas reddish in the lumen with large number of filiform white parasites from 1 to $1.5 \mathrm{~cm}$ in length, morphologically compatible with Heterakis gallinarum. The liver showed large and circular, multifocal, whitish areas that extended towards the parenchyma. Histologically, was observed marked transmural typhlitis, severe proliferation of fibroblasts with multifocal neovascularization, histiocytic infiltrate, giant cell enlargement, bacterial myriads, presence of eosinophilic circulars trophozoites, with 6-20 $\mu \mathrm{m}$ in diameter, morphologically compatible with Histomonas meleagridis. These structures were positive in PAS staining. In the liver, a lesion was characterized by aleatory necrotizing hepatitis with abundant macrophagic and heterophilic infiltrate and some lymphocytes, as well as several foci of necrosis associated with numerous parasitis, intralesional, weakly eosinophilic, PAS-positive structures similar to those observed cecum.

Discussion: All aspects observed in this outbreak were characteristic of Histomonas meleagridis infection. This protozoal infection occurs predominantly in turkeys, with high rates of morbidity and mortality, however, can affect chickens, chukar partridges, pheasants and peacocks. Histomoniasis occurs after ingestion of nematode eggs present in the feces of birds and earthworms. In addition, to the intestine and liver, there may be systemic parasitism with lesions in other organs such as kidneys, lungs, spleen and cloacal sac. Overcrowding of animals, the creation and use of chickens to hatch peacocks eggs, as well as the poor hygiene conditions were the main risk factors for the development of the disease. Due to the absence of specific clinical signs, the disease can be easily confused with other disorders. macroscopic and microscopic examinations are recommended to assist in confirming the definitive diagnosis of histomoniasis. Thus, it can be said that H. meleagridis may be cause of mortality in peacocks (Pavo cristatus) created in free system in Rio Grande do Sul, State. The diagnosis of this condition should be considered in cases of mortality in exotic bird breeding, especially when created together with most resistant species such as chickens and other poultry.
\end{abstract}

Keywords: protozoan disease, enterohepatitis, histomoniasis, peacock.

Descritores: protozoário, enterohepatite, histomoniase, pavão. 


\section{INTRODUÇÃO}

Histomoníase também conhecida por enterohepatite, tiflohepatite ou "blackhead" (cabeça negra) é uma doença causada pelo protozoário Histomonas meleagridis (filo Parabasalia, classe Tritrichomonadea, ordem Tritrichomonadida, familia Dientamoebidae/Protrichomonadinae) [8,15,24]. Dentre as espécies mais afetadas, os perus são os que apresentam maior morbidade e mortalidade $[9,20,23]$. Entretanto, são descritos casos em galinhas e aves exóticas $[3,15,18,21]$.

Essa infeção é a segunda doença mais importante causada por protozoários em aves domésticas, atrás apenas da coccidiose, doença de alto impacto econômico que acomete aves de todos os continentes [5]. A enfermidade é causadora de grandes prejuízos econômicos a criadores de aves, tendo em vista o decréscimo na produção, a desuniformidade dos lotes, os gastos com medicamentos e controle dos surtos [15]. Tais prejuízos elevam a necessidade de maior atenção quanto ao monitoramento de sua ocorrência em criações de aves, especialmente em países como o Brasil, que atualmente se consagra como o segundo maior produtor e maior exportador de carne de frango do mundo [6]. O objetivo desse relato é descrever um surto de histomoníase em pavões de um criadouro doméstico no oeste do Rio Grande do Sul.

\section{CASO}

Foi registrado um surto de mortalidade de pavões (Pavo cristatus) em uma propriedade rural no munícipio de Uruguaiana, no oeste do Rio Grande do Sul. Durante a visita ao estabelecimento foram avaliados os aspectos epidemiológicos e os sinais clínicos apresentados pelas aves. O surto ocorreu em um lote de 12 pavões criados em conjunto com galinhas domésticas (Gallus gallus). Desse total 8 pavões, com aproximadamente 2 meses de idade, apresentaram sinais clínicos caracterizados por apatia, cabeça caída e arroxeada, olhos fechados, anorexia e fezes amareladas ou esbranquiçadas que culminaram com a morte. Segundo o proprietário, os ovos de pavões eram chocados por galinhas da propriedade.

Uma fêmea jovem foi submetida à necropsia $\mathrm{e}$ amostras de diversos órgãos foram fixados em formalina a $10 \%$. Macroscopicamente, a parede do ceco estava acentuadamente espessada e substituída por material friável, amorfo, branco amarelado com áreas marrons a avermelhadas (Figura 1). Havia ainda abundante quantidade de parasitos nematoides, brancacentos, alongados com 8 a $13 \mathrm{~mm}$ de comprimento e extremidades afiladas, morfologicamente compatíveis com Heterakis gallinarum.

O fígado estava aumentado, com superfície capsular levemente irregular. Foram observadas também áreas multifocais bem delimitadas com até $1 \mathrm{~cm}$ de diâmetro, branco-amareladas e com centro levemente deprimido. Ao corte essas lesões se estendiam mais profundamente no parênquima (Figura 2).

As amostras foram processadas para histopatologia e lâminas com cortes de $4 \mu \mathrm{m}$ foram confeccionadas e coradas pela técnica de hematoxilinaeosina (HE). Fragmentos de ceco e do fígado foram submetidos ainda à coloração pelo Ácido Periódico de Schiff (PAS) e Grocott.

Microscopicamente, foi observada no ceco intensa deposição de material fibrinonecrótico com formação de aglomerado bem delimitado de macrófagos e heterófilos íntegros e degenerados que se estendia da mucosa até a serosa em algumas áreas do ceco caracterizando tiflite transmural acentuada. Havia intensa proliferação de fibroblastos com formação de uma matriz conjuntiva frouxa com neovascularização multifocal, infiltrado macrofágico, eventuais células gigantes, miríades bacterianas e presença de trofozoítos redondos a ovais eosinofílicos, de 6-20 $\mu \mathrm{m}$ de diâmetro, morfologicamente compatíveis com Histomonas meleagridis.

O fígado apresentava extensas áreas multifocais de necrose e intenso infiltrado inflamatório rico em macrófagos, heterófilos e alguns linfócitos. Foram observadas ainda múltiplas áreas aleatórias de necrose e inúmeras estruturas parasitárias intralesionais redondas ou ovais com 10-20 $\mu \mathrm{m}$ e levemente eosinofílicas (Figura 3). Observam-se ainda ocasionais núcleos levemente basofílicos com 2-5 $\mu \mathrm{m}$ de diâmetro, bem delimitados por um halo claro, compatíveis com o protozoário Histomonas meleagridis. Tanto as seções do ceco como do fígado demonstram lesões associadas a protozoários PAS-positivas (Figura 4).

Foram observados também inúmeros exemplares de nematódeos morfologicamente compatíveis com Heterakis gallinarum em galinhas abatidas na propriedade.

\section{DISCUSSÃO}

Os achados macroscópicos e microscópicos observados nesse pavão foram compatíveis com tiflite e 


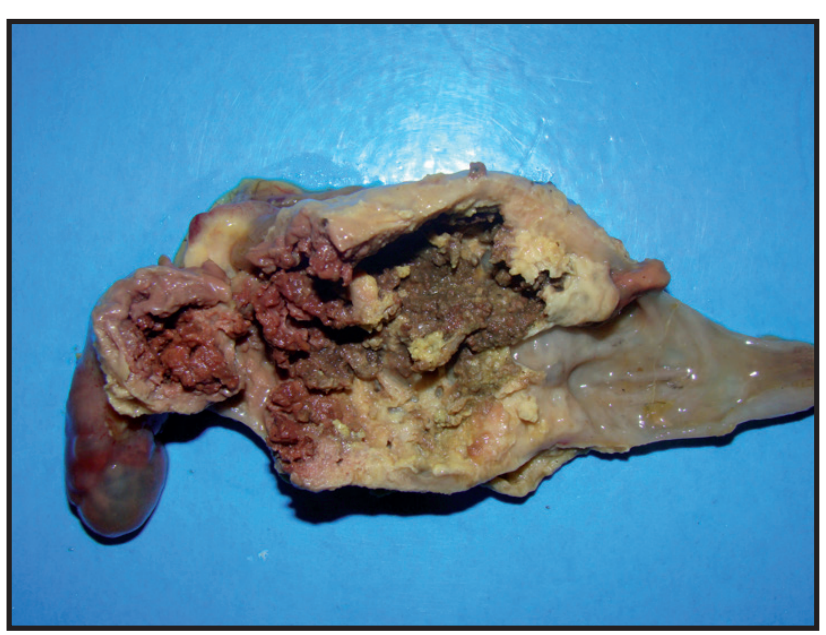

Figura 1. Ceco. Intenso espessamento a parede com irregularidade da serosa com abundante material fibrinoide friável, amorfo, amarronzado e com áreas de hemorragia.

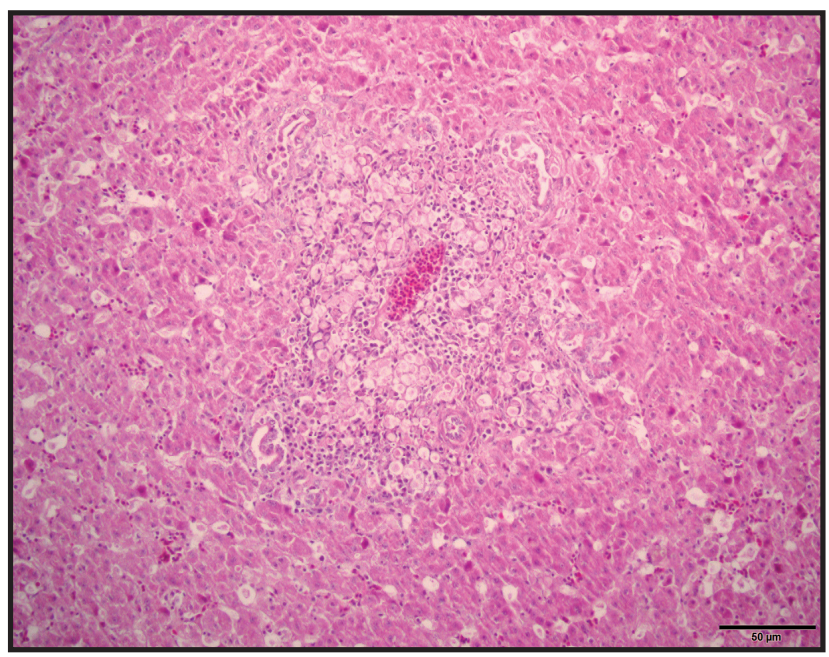

Figura 3. Fígado. Área focal de necrose com infiltrado inflamatório predominantemente composto por macrófagos associado a trofozoítos morfologicamente compatíveis com Histomonas meleagridis.

hepatite necrótica associadas à infecção por Histomonas meleagridis [2,3,9,12,14,24]. A histomoníase afeta diversas espécies de aves domésticas e silvestres $[1,14,15,23,24]$. Dentre as espécies afetadas, os perus são os que apresentam maior morbidade e mortalidade [7,14], entretanto também pode acometer galinhas [1], perdizes chukar [18], faisões e pavões [17,24].

O protozoário Histomonas meleagridis na fase não ameboide apresenta-se esférico, com cerca de $10 \mu \mathrm{m}$ de diâmetro e pode apresentar ou não flagelo. Quando o mesmo se encontra no lúmen cecal das aves possui um único flagelo com até 11 $\mu \mathrm{m}$ de comprimento que eventualmente apresenta vacúolos citoplasmáticos e grânulos [10]. As formas encontradas nos tecidos são aflageladas e com três diferentes formatos, todas elas observadas nas amostras avaliadas, embora histologicamente não seja

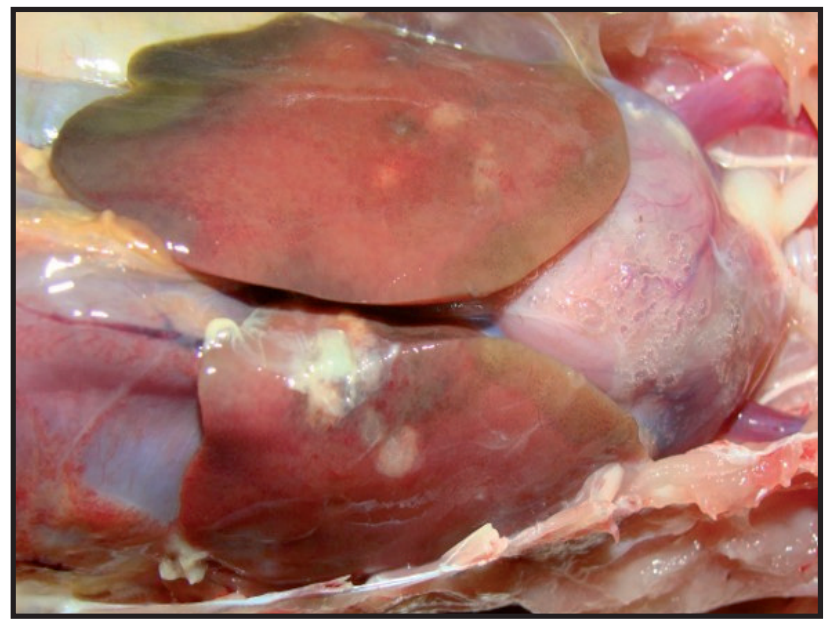

Figura 2. Fígado. Superfície capsular com áreas circulares multifocais, bem delimitadas, branco amarelada.

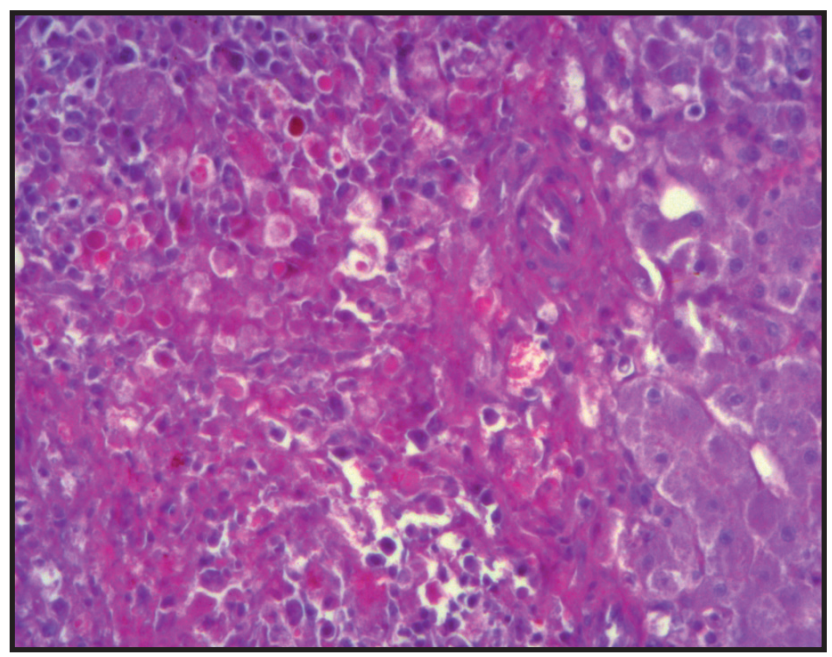

Figura 4. Fígado. Trofozoítos de Histomonas meleagridis PAS-positivos em meio a hepatócitos necróticos. [PAS, 20x].

fácil a diferenciação. Essas formas parasitárias são denominadas de invasiva, encontrando-se na periferia das lesões, a vegetativa que são mais numerosas e a resistente, que se encontra nas porções mais antigas das lesões e são um pouco menores [15].

Tanto na pavoa necropsiada como em galinhas da propriedade notou-se grande quantidade de exemplares de $H$. gallinarum. Para completar o ciclo de vida, o protozoário necessita de um hospedeiro intermediário, estando frequentemente associado a nematódeos do gênero Heterakis que também pode servir como hospedeiro paratênico, servindo esporadicamente como proteção para os trofozoítos contra as alterações de $\mathrm{pH}$ do organismo da ave e ou do próprio ambiente $[2,9,15]$.

A enfermidade desenvolve-se decorrente da ingestão de ovos do nematódeo presentes nas fezes de aves contaminadas ou de minhocas $[5,15,20]$. No 
ceco da ave infectada, os trofozoítos são liberados a partir dos ovos de $H$. gallinarum, que penetram na parede do ceco e perdem o flagelo passando ao estágio ameboide [8] na qual se aderem à parede cecal e, por via hematógena, se disseminam para o fígado causando lesões características da doença como hepatite necrótica, em média, seis dias a partir da infecção $[11,16]$. Nesse surto, essa rota foi provavelmente a mais importante para a alta infecção nos pavões.

A criação conjunta dos pavões com aves resistentes (galinhas) contribuiu para uma alta contaminação do agente no sistema de criação e as escassas condições de biosseguridade contribuíram para a alta morbidade e mortalidade da espécie mais sensível. A associação da grande lotação de animais, criação e utilização de galinhas para chocar os ovos dos pavões provavelmente foi principal fonte de infecção de $H$. meleagridis.

A tiflite necrótica é primária e foi uma das principais lesões observadas na necropsia. Essa lesão é decorrente da eclosão dos ovos do H. gallinarum, que se fixam na parede do órgão produzindo inflamação acentuada, com espessamento de parede, associada à heterófilos que formam o material amarelo-acastanhado multinodular à coalescente visualizado na luz do órgão $[11,14]$. Já a lesão hepática observada é secundária e ocorre pela disseminação do agente a partir do ceco, podendo levar a um quadro de insuficiência hepática grave $[11,16]$.

Devido à ausência de sinais clínicos específicos da enfermidade, o exame de necropsia é a principal ferramenta de diagnóstico de infecção por $H$. meleagridis $[13,14]$ que, associado à histologia torna possível o estabelecimento do diagnóstico preciso.

Além do ceco e do fígado, ocasionalmente pode haver parasitismo de forma sistêmica, tendo relatos de lesões nos rins, pulmões, baço e bolsa cloacal $[4,17,22]$ o que não foi observado neste caso.

Apesar dos sinais clínicos de apatia, emagrecimento e anorexia serem semelhantes aos apresentados por diversos outros distúrbios, as lesões observadas na microscopia são suficientes para estabelecer o diagnóstico definitivo da doença, diferenciando a histomoníase de outras condições como leucose, infecções bacterianas, micoses e coccidiose [14] que podem apresentar características epidemiológicas e clínico-patológicas bastante semelhantes.

No Brasil, há poucos relatos da doença em pavões [17,24], no entanto essa condição deve ser incluída como causa de mortalidade para animais dessa espécie e produtores devem estar atentos para sua ocorrência especialmente em aves jovens e criadas em consórcio com outras espécies galináceas mais resistentes.

Uma vez estabelecido o diagnóstico, foi recomendado ao proprietário medidas de bioseguridade, proteção dos comedouros e bebedouros de contaminação fecal, separação dos lotes de galinhas e pavões, pois estas são mais resistentes e apresentam forma subclínica da doença com baixa mortalidade e atuam como reservatório de $H$. gallinarum e $H$. meleagridis [19].

Dessa forma, pode-se afirmar que $H$. meleagridis pode ser causa de mortalidade em pavões (Pavo cristatus) criados em sistema livre no Rio Grande do Sul. O diagnóstico dessa condição deve ser considerado em casos de mortalidade em criações de aves exóticas, especialmente quando criadas em consórcio com espécies mais resistentes como galinhas e outras aves domésticas.

Declaration of interest. The authors report no conflicts of interest. The authors alone are responsible for the content and writing of the paper.

\section{REFERENCES}

1 Araújo J.L., Olinda R.G., Frade M.T.S., Maia L.A. \& Dantas A.F.M.D. 2015. Histomoniasis outbreak in free-range chickens in semiarid Paraíba, Brazil. Semina Ciências Agrárias. 36(1): 307-317.

2 Boralli I.C., Alves R.M., Coelho Neto E. \& Neves M.F. 2008. Histomonose. Revista Científica Eletrônica de Medicina Veterinária. 7(11): 1-5.

3 Clarke L.L., Beckstead R.B., Hayes J.R. \& Rissi D.R. 2017. Pathologic and molecular characterization of histomoniasis in peafowl (Pavo cristatus). Journal of Veterinary Diagnostic investigation. 29(2): 237-241.

4 Cortes P.L., Chin R.P., Bland M.C., Crespo R. \& Shivaprasad H.L. 2004. Histomoniasis in the Bursa of Fabricius of Chickens. Avian diseases. 48(3): 711-715.

5 Dolka B., Zbikowski A., Dolka I. \& Szeleszczuk P. 2015. Histomonosis - na existing problem in chicken flocks in Poland. Veterinary Research Communications. 39(3): 189-195. 
6 Embrapa. 2017. Estatística mundial frango de corte. Disponivel em $<$ https://www.embrapa.br/suinos-e-aves/cias/ estatisticas/frangos/mundo>. [Accessed online in April 2018].

7 Hafez M.H. 2009. Doenças entéricas em perus In: Revolledo L. \& Ferreira A.J.P. (Eds). Patologia Aviária. Barueri: Manole, pp.480-491.

8 Hauck R. \& Hafez M.H. 2013. Experimental infections with the protozoan parasite Histomonas meleagridis: a review. Parasitology Research. 112(1): 19-34.

9 Hess M., Liebhart D., Bilic I. \& Ganas P. 2015. Histomonas meleagridis - New insights into an old pathogen. Veterinary Parasitology. 208(1-2): 67-76.

10 Honingberg B.M. \& Bennet C.J. 1971. Lightmicroscopic Observations on Structure and Division of Histomonas meleagridis (Smith). The Journal of Protozoology. 18(4): 687-987.

11 Huber K., Reynaud M.C., Callait M.P. \& Zenner L. 2006. Histomonas meleagridis in turkeys: dissemination kinetics in host tissues after cloacal infection. Poultry Science. 85(6): 1008-1014.

12 Jowett W. 1911. Infectious entero-hepatitis or typhlo-hepatitis. A disease of young turkeys. Journal of Comparative Pathology and Therapeutics. 24(4): 198-302.

13 Kemp R.L. \& Reid W.M. 1966. Staining techniques for differential diagnosis of histomoniasis and mycosis in domestic poultry. Avian Diseases. 10(3): 357-363.

14 Mcdougald L.R. 2005. Blackhead Disease (Histomoniasis) in Poultry: A Critical Review. Avian Disease. 49(4): 462476.

15 Mcdougald L.R. 2008. Histomoniasis (Blackhead) and other Protozoan of the Intestinal Tract. In: Saif Y.M. (Ed). Diseases of Poultry. 12th edn. Ames: Blackwell, pp.1067-1221.

16 McGuire W.C. \& Morehouse N.F. 1958. Blood-induced blackhead. Journal of Parasitology. 44(3): 292-296.

17 Michelazzo M.M.Z., Sasse J.P., de Souza M., Marutani V.H.B., Sampaio Baptista A.A., Garcia J.L., Alfieri A.A. \& Headley S.A. 2017. Systemic histomoniasis in a Leucistic Indian Peafowl (Pavo cristatus) from Southern Brazil. Avian diseases. 61(3): 325-329.

18 Oliveira L.G.S., Boabaid F.M., Lorenzett M.P., Rolim V., Santos H.F., Driemeier D. \& Cruz C.E.F. 2017. Outbreaks of mycoplasmosis and histomoniasis in a southern Brazilian flock of ornamental birds. Acta Scientiae Veterinariae. 45(1): $1-5$.

19 Popp C., Hauck R., Balczulat S. \& Hafez H.M. 2011. Recurring histomonosis on an organic farm. Avian Diseases. 55(2): 328-330.

20 Reid W.M. 1967. Etiology and dissemination of the blackhead disease syndrome in turkeys and chickens. Experimental Parasitology. 21(2): 249-275.

21 Reis J.L., Beckstead R.B., Brown C.C. \& Gerhold R.W. 2009. Histomonas meleagridis and capillarid infection in a captive chukar (Alectoris chukar). Avian Diseases. 53(4): 637-639.

22 Sentíes-Cué G., Chin R.P. \& Shivaprasad H.L. 2009. Systemic histomoniasis associated with high mortality and unusual lesions in the bursa of Fabricius, kidneys, and lungs in commercial turkeys. Avian Diseases. 53(2): 231-238.

23 Silva T.M. Okamoto A.S., Smaniotto B.D., Paes A.C. \& Andreatti Filho R.L. 2014. Histomoníase em peru (Meleagris gallopavo) - relato de caso. Veterinária e Zootecnia. 21(2): 269-274.

24 Trindade M.M., Schneiders G.H., Corrêa I.M.O., Flores F., Lovato M. \& Kommers G. D. 2011. Histomoníase em pavão (Pavo cristatus). A Hora Veterinária. 31(184): 56-58. 